\title{
Continuous glucose monitoring for hypoglycaemia in children: Perspectives in 2020
}

DOI:

10.1111/pedi.13029

\section{Document Version}

Accepted author manuscript

Link to publication record in Manchester Research Explorer

\section{Citation for published version (APA):}

Worth, C., Dunne, M., Ghosh, A., Harper, S., \& Banerjee, I. (2020). Continuous glucose monitoring for hypoglycaemia in children: Perspectives in 2020. Pediatric Diabetes, 21(5), 697-706.

https://doi.org/10.1111/pedi.13029

\section{Published in:}

Pediatric Diabetes

\section{Citing this paper}

Please note that where the full-text provided on Manchester Research Explorer is the Author Accepted Manuscript or Proof version this may differ from the final Published version. If citing, it is advised that you check and use the publisher's definitive version.

\section{General rights}

Copyright and moral rights for the publications made accessible in the Research Explorer are retained by the authors and/or other copyright owners and it is a condition of accessing publications that users recognise and abide by the legal requirements associated with these rights.

\section{Takedown policy}

If you believe that this document breaches copyright please refer to the University of Manchester's Takedown Procedures [http://man.ac.uk/04Y6Bo] or contact uml.scholarlycommunications@manchester.ac.uk providing relevant details, so we can investigate your claim.

\section{OPEN ACCESS}




\title{
Continuous Glucose Monitoring for Hypoglycaemia in Children: Perspectives in 2020
}

\author{
Chris Worth ${ }^{1}$, Mark Dunne ${ }^{2}$, Arunabha Ghosh ${ }^{3}$, Simon Harper ${ }^{4}$, Indraneel \\ Banerjee $^{1}$ \\ ${ }^{1}$ Department of Paediatric Endocrinology, Royal Manchester Children's Hospital, Manchester, UK \\ ${ }^{2}$ Faculty of Biology, Medicine and Health, University of Manchester, Manchester, UK \\ ${ }^{3}$ Department of Inherited Metabolic Disease, St Mary's Hospital, Manchester, UK \\ ${ }^{4}$ Faculty of Computer Engineering, University of Manchester, Manchester, UK
}

\section{Corresponding author}

Chris Worth

Department of Paediatric Endocrinology

Royal Manchester Children's Hospital

Oxford Road

Manchester

M13 9WL

\section{Funding statement}

No funding was received by any member of the authorship. Both Dexcom Inc and Congenital Hyperinsulinism International have provided Continuous Glucose Monitoring devices as a donation in kind to the Northern Congenital Hyperinsulinism Service based at Royal Manchester Children's Hospital for whom Drs Worth and Banerjee work. Research in our laboratories is supported by the Northern Congenital Hyperinsulinism (NORCHI) charitable fund, by the Manchester Academic Health Sciences Centre and by The University of Manchester MRC Confidence in Concept (CiC) Award (MC_PC_18056)

\section{Conflict of interest disclosure}

All authors declare no conflict of interest.

\section{Ethics approval statement}

This is not required as this manuscript is purely a review of the literature.

\section{Acknowledgements}

We would like to thank Lynne Calvert at Threefold Creative Ltd for her assistance in creating our figures.

\section{Author contributions}

All authors were involved in drafting the manuscript and all read and approved the final version before submission.

\section{Key words}

Continuous Glucose Monitoring, Hypoglycaemia, Children, Hyperinsulinism, Machine Learning 


\section{Abstract}

Hypoglycaemia in children is a major risk factor for adverse neurodevelopment with rates as high as $50 \%$ in hyperinsulinaemic hypoglycaemia $(\mathrm{HH})$. A key part of management relies upon timely identification and treatment of hypoglycaemia. The current standard of care for glucose monitoring is by infrequent fingerprick plasma glucose testing but this carries a high risk of missed hypoglycaemia identification. High-frequency Continuous Glucose Monitoring (CGM) offers an attractive alternative for glucose trend monitoring and glycaemic phenotyping but its utility remains largely unestablished in disorders of hypoglycaemia. Attempts to determine accuracy through correlation with plasma glucose measurements using conventional methods such as Mean Absolute Relative Difference (MARD) overestimate accuracy at hypoglycaemia. The inaccuracy of CGM in true hypoglycaemia is amplified by calibration algorithms that prioritise hyperglycaemia over hypoglycaemia with minimal objective evidence of efficacy in $\mathrm{HH}$. Conversely, alternative algorithm design has significant potential for predicting hypoglycaemia to prevent neuroglycopaenia and consequent brain dysfunction in childhood disorders. Delays in the detection of hypoglycaemia, alarm fatigue, device calibration and current high cost are all barriers to the wider adoption of CGM in disorders of hypoglycaemia. However, machine learning, artificial intelligence and other computer-generated algorithms now offer significant potential for further improvement in CGM device technology and widespread application in childhood hypoglycaemia. 


\section{Background}

Home testing of glucose was first made available to patients in 1925 by boiling a sample of urine with chemical reagents on the stove. Fortunately, steady progress was made throughout the $20^{\text {th }}$ century with the development of Clinistix and Dextrostix to dipstick test urine and blood respectively ${ }^{1}$. In 1979 Karam et al described their use of an early form of Continuous Glucose Monitoring (CGM) linked to a Dextrose infusion during insulinoma resection ${ }^{2}$. However, progress with CGM stalled until 1999 when the Medtronic MiniMed Continuous Glucose Monitoring system was released. This allowed patients with Type 1 Diabetes Mellitus (T1DM) to monitor glucose profiles at home, retrospectively view them with their doctor and adjust treatment accordingly ${ }^{3}$. Rapid progress has been made over the last two decades with several devices now available for use in patients with diabetes (Table 1) including non-adjunctive devices wearable for 10 days with no requirement for calibration ${ }^{4}$.

Modern CGM devices consist of a wearable sensor that automatically measures glucose in interstitial fluid (ISF) several times a minute and reports an average every few minutes to a wireless receiver nearby. ISF glucose reacts with an enzymatic technology to liberate electrons and generate an electric current. Via a continuously shifting algorithm, this electrical current is converted to a blood glucose value ${ }^{5}$. This algorithm is finetuned via patient or factory calibration to ensure accuracy over the recording periods ${ }^{6}$ (Figure 1 ).

CGM devices are currently only licensed for patients with diabetes and most studies have targeted a reduction in $\mathrm{HbA} 1 \mathrm{C}$ rather than hypoglycaemia episodes as a clinical end point. In this paper, we review the evidence available for the use of CGM in hypoglycaemia disorders and discuss future developments in this field. 


\section{The problem of hypoglycaemia}

Recurrent episodes of hypoglycaemia lead to inadequate cerebral glucose supply. In the developing brain of neonates and infants, this can lead to serious long-term neurological impairments ranging from mild neurocognitive dysfunction to epilepsy, hemiparesis and severe mental retardation?. Several studies have demonstrated the significant impact of neonatal hypoglycaemia on long-term neurodevelopmental outcomes ${ }^{8,9}$ with evidence of persistent MRI changes in these patients ${ }^{10}$. Hypoglycaemia secondary to hyperinsulinism is even more devastating, with almost $50 \%$ of children demonstrating neurological impairments ${ }^{11}$ regardless of the persistence of disease $\mathrm{e}^{12}$. Hypoglycaemia is difficult to predict in $\mathrm{HH}$ as insulin secretion is dysregulated, and recurrent hypoglycaemia blunts the clinical signs and counter-regulatory responses, resulting in unpredictable and often silent hypoglycaemia episodes ${ }^{13}$. While this paper concentrates mainly on non-diabetic disorders of hypoglycaemia it is worth noting the potentially devastating impact of hypoglycaemia on those with diabetes sometimes leading to "dead in bed" syndrome ${ }^{14}$. The cornerstone of management is the timely identification and treatment of such episodes. The current standard of care is patient observation and intermittent fingerpick testing, but this provides no details of trends and risks missing hypoglycaemia between infrequent tests. CGM provides a highly attractive alternative to monitor glucose trends and reduce the risk of hypoglycaemia events, but current evidence is scant in relation to both accuracy and efficacy in hypoglycaemia disorders in children.

\section{Accuracy of CGM at hypoglycaemia}

Accuracy of CGM devices is described as either analytical or clinical. Clinical accuracy is based on the mean accuracy of performance or the number of outliers ${ }^{5}$. Analytical accuracy can be reported as either trend or point accuracy, of which Mean Absolute Relative Difference (MARD) is the most commonly reported measure ${ }^{5}$. MARD is the average of the absolute error between all CGM values and is routinely calculated and reported as a key metric by manufacturers. 
Despite significant improvements in CGM since first-generation devices ${ }^{15}$, accuracy is still not clinically acceptable at hypoglycaemia values ${ }^{16,17}$ and more so in non-diabetic patients: The best published MARD value in neonates at hypoglycaemia is $16 \%{ }^{18}$ with only $86 \%$ of values in Zone $A$ (allowing clinically correct decisions) on a Modified Clarke Error Grid (MCEG) ${ }^{19}$ (Figure 2). In the clinical setting these values are suboptimal when compared to manufacturers figures for patients with diabetes ${ }^{20,21}$. Importantly, under controlled conditions elevated insulin has been reported to increase the discrepancy between recorded ISF glucose and plasma glucose in healthy volunteers and therefore further worsens the accuracy of hypoglycaemia-detection ${ }^{22}$. The mechanism underlying this is has been suggested to be related to enhanced uptake of interstitial glucose by adipocytes that is not fully compensated for by increased delivery of glucose from the microcirculation ${ }^{22}$. In patients with $\mathrm{HH}$, these observations are highly relevant to the accuracy of CGM in reporting hypoglycaemia as default profiles will over-report incidences of low glucose levels.

While MARD is the most commonly reported measure of accuracy, it is a cross-sectional value and does not account for the benefits of trend direction and rate of change that CGM can provide ${ }^{5}$. MARD is reported from clinical trial data but this represents the accuracy of the system within that context and should not be understood as a precise value to be used outside of the trial ${ }^{23}$. In practice, this means that from a single MARD value it is not possible to ensure meter accuracy will meet ISO criteria, but it is highly likely if MARD falls between 3.25 and $5.25 \%{ }^{24}$. MARD is calculated by the sensitivity and specificity of values but without reference to the timing of hypoglycaemia alarms. If predictive alarms occur too early or late this will result in false positive or negative errors, thereby impairing the detection of clinical hypoglycaemia ${ }^{25}$. In contrast to MARD, a more useful report of accuracy for a hypoglycaemia device is the false positive and negative rate as this will provide an indication of how effectively the device can alert the user to impending hypoglycaemia. A useful device should have false negative and positive rates of $<5 \%$ and $<10 \%$ respectively and will typically require a MARD of $<7.5 \%$ to achieve this ${ }^{25}$. Using such criteria, accuracy in reported studies are well short of expected standards with a false positive rate of $60 \%$ in neonates ${ }^{26}$ and a false negative rate of $35 \%$ in $\mathrm{HH}^{27}$ detracting from the confidence of use in clinical practice. 
The accuracy of a CGM device is intrinsically linked to the calibration algorithm used to convert the raw electrical signal to a meaningful plasma glucose value. The accuracy of CGM at hypoglycaemia values is particularly affected by the calibration algorithm ${ }^{28}$. If calibration is performed at a time of rapid glucose change (as is common in patients with dysregulated insulin secretion and wide glucose variability in $\mathrm{HH}$ and Glycogen Storage Disease) algorithm performance is seriously compromised ${ }^{5}$. Conversely, MARD is significantly improved if calibration glucose values are as widely spaced as possible ${ }^{29}$.

Accuracy of CGM can also be improved through the alteration of associated algorithms. Guerra et al and Zavitsanou et al both showed an improvement in CGM accuracy using an online, automatically updating algorithm ${ }^{30,31}$. Acciaroli et al demonstrated that a reduction in the frequency of calibrations from once a day to no calibration caused only a small deterioration in accuracy when using an automatically updating algorithm ${ }^{32}$. Personalisation of calibration algorithms further improves accuracy; King et al reported a Mean Absolute Difference of $24.5 \mathrm{mg} / \mathrm{dL}$ during hypoglycaemia with raw sensor data but demonstrated an improvement to $11.5 \mathrm{mg} / \mathrm{dL}$ with modelling of group interstitial glucose dynamics and to $10.4 \mathrm{mg} / \mathrm{dL}$ when individual interstitial glucose dynamics were used. Finally, accuracy is improved when calibration is performed within the setting it is trying to detect. Monsod et al demonstrated no significant difference between sensor and plasma glucose levels when calibration was performed during hyperinsulinaemic, hypoglycaemic conditions ${ }^{22}$. These successes demonstrate the potential of algorithm personalisation for improvement of hypoglycaemia prediction and application potential for childhood hypoglycaemia.

\section{Efficacy of CGM for detection and prevention of hypoglycaemia}

\section{Diabetes}

Early trials of CGM in patients with diabetes showed a good reduction in $\mathrm{HbA}_{1} \mathrm{C}^{33,34}$ but did not demonstrate the expected ${ }^{35}$ reductions in hypoglycaemia incidence ${ }^{36}$. This may have been because trials were designed to reduce $\mathrm{HbA} 1 \mathrm{C}$ rather than time in hypoglycaemia. More recently, trials 
specifically investigating hypoglycaemia in patients with diabetes have shown good improvements ${ }^{37}$, particularly overnight ${ }^{38}$ and when using predictive alarms ${ }^{39}$.

However, CGM devices have been designed for use in patients with diabetes where there is an established relationship between plasma glucose, insulin administration and carbohydrate intake. Despite sound theoretical reasons to anticipate benefit from CGM devices, their utility and efficacy in other conditions of hypoglycaemia cannot be assumed.

\section{Neonates}

Several studies have investigated the use of CGM in neonatal intensive care and found it well tolerated ${ }^{40}$. Reducing painful procedures results in improved cognitive and motor function outcomes ${ }^{41}$ and is facilitated by CGM. Between $25 \%-80 \%$ of neonates demonstrated hypoglycaemia on CGM that had gone undetected by heelprick glucose monitoring ${ }^{42,43}$. However, despite CGM also reducing the duration of hypoglycaemia episodes ${ }^{44}$, the long-term clinical benefit is uncertain as undetected hypoglycaemia was not associated with worse neurological outcomes at follow up at two years of age $e^{45}$.

\section{GSD}

Patients with Glycogen Storage Diseases (GSDs) are at risk of significant hypoglycaemia when exposed to prolonged fasting. Euglycaemia is maintained through regular meals, the provision of uncooked cornstarch, and often continuous overnight feeding, usually via gastrostomy ${ }^{46}$. Several studies have demonstrated the utility of CGM in detecting unsuspected hypoglycaemia, particularly overnight $^{47,48}$. This allows for the manipulation of diet and treatment and, in GSD I, has been shown to be associated with reduced time in hypoglycaemia, reduction in liver size and improvement in biochemical markers of disease control ${ }^{49}$. Further studies have since demonstrated improved detection of both hypoglycaemia and hyperglycaemia in patients with GSD I, III and IX ${ }^{50}$. Intermittent use of CGM for glucose profiling in GSD patients is now standard practice in many centres as it provides a more accurate picture of a patient's daily profile than is obtainable from an inpatient stay. This has proven to be popular with families and has shown not only an improvement in markers 
of disease severity but also increased adherence with medication and improved patient quality of life ${ }^{51}$.

\section{Adrenal Insufficiency}

Hypoglycaemia is considered a rare problem for adults with adrenal insufficiency (Al) ${ }^{52}$. However, in those with morning headache or discomfort, overnight CGM revealed hypoglycaemia in 5 out of 6 patients who had all demonstrated normal morning fingerprick glucose testing ${ }^{53}$. Increasing hydrocortisone dosing not only prevented overnight hypoglycaemia but also resulted in resolution of symptoms $s^{53}$.

Hypoglycaemia is a more frequent problem in children with Al but is still difficult to detect on routine monitoring. Cambiaso et al studied 11 children with Al and Growth Hormone Deficiency (GHD) who were normoglycaemic on routine testing. CGM revealed severe hypoglycaemia $(<2.7 \mathrm{mmol} / \mathrm{L})$ overnight in three children, with time in hypoglycaemia ranging from 30 to 150 minutes ${ }^{54}$. Those with hypoglycaemia had a lower daily dose of hydrocortisone (5.9 vs $8.5 \mathrm{mg} / \mathrm{m} 2 /$ day, $\mathrm{p}=0.04$ ) and repeat testing after dose increase revealed resolution of hypoglycaemia ${ }^{54}$. This study demonstrates the added utility of CGM in detecting hypoglycaemia that would have otherwise been overlooked.

\section{Hyperinsulinism}

Hypoglycaemia in children with hyperinsulinism is not only unpredictable and often profound but is accompanied by the suppression of alternative fuels and a counterregulatory response. Therefore, children with $\mathrm{HH}$ are at an unacceptably high risk of brain injury ${ }^{55}$ irrespective of the permanence of their disease ${ }^{12}$. It is well established that the severity of hypoglycaemia in $\mathrm{HH}$ determines the extent of brain injury; by contrast, reducing the depth and frequency of hypoglycaemia may protect the vulnerable brain in neonates and infants from the irreversible effects of neuroglycopaenia.

There have been no long-term studies on the use of CGM in children with hyperinsulinism to demonstrate unequivocal benefit. Anecdotal reports and case studies have commented on the usefulness of CGM in categorising the extent and degree of hypoglycaemia in children with $\mathrm{HH}^{56}$. The 
very poor accuracy in this patient group ${ }^{27}$, may be due to increased discrepancies between plasma and sensor glucose at hyperinsulinaemia ${ }^{22}$. Despite this, CGM allows for a dynamic view of hypoglycaemia and enables clinicians to measure responses to treatment ${ }^{27,57}$. Alsaffar et al used a factory-calibrated flash CGM device in a small group of children with $\mathrm{HH}$ and reported disappointing hypoglycaemia performance ${ }^{58}$. As in previous studies ${ }^{56}$, parents found glucose trends helpful but were not confident in relying upon CGM glucose levels alone in the regular monitoring of blood glucose $e^{58}$.

In adult patients with $\mathrm{HH}, 28 \%$ of those studied had asymptomatic hypoglycaemia detected by CGM and required subsequent treatment alterations ${ }^{59}$. In adult patients with hypoglycaemia secondary to insulinoma, CGM also identified significant hypoglycaemia in patients who were thought to be normoglycaemic. One patient was found to be severely hypoglycaemic $(<2.2 \mathrm{mmol} / \mathrm{L})$ for $48 \%$ of the monitoring period with complete resolution following therapy with Diazoxide ${ }^{60}$. The authors of this study acknowledged the significant impact that CGM had upon the treatment and monitoring of their patients and its superiority over fingerprick glucose monitoring. Similar results have been reproduced elsewhere; Sawyer et al demonstrated a reduction in the number of hypoglycaemic events and visits to the hospital for one patient with insulinoma after use of $\mathrm{CGM}^{61}$. Multiple centres have demonstrated the utility of CGM for diagnosis and surgical monitoring of patients with insulinomas $\mathrm{s}^{62,63}$ and to detect hypoglycaemia in adult patients following bariatric surgery ${ }^{64,65}$.

\section{Acute illness in low-income settings}

In low-income countries, paediatric hypoglycaemia is a life-threatening complication of many common diseases such as malaria and malnutrition ${ }^{66}$. In sub-Saharan Africa, up to $7 \%$ of all hospital admissions are complicated by hypoglycaemia with an associated increase in the mortality rate from $4 \%$ to $20 \%{ }^{67}$. Malaria is accompanied by hypoglycaemia in up to $25 \%$ of cases ${ }^{68}$ with an increase in the case fatality rate to up to $64 \%$ in those with hypoglycaemia ${ }^{69}$. This hypoglycaemia prevalence is likely to be an underestimate as most hospitals in this setting use a single glucose test on admission as their screening and therefore risk missing inpatient hypoglycaemia ${ }^{66}$. In a study of children with 
malaria in Mozambique CGM demonstrated significantly more hypoglycaemia than previously thought and allowed better monitoring of severely ill patients at risk of life-threatening hypoglycaemia ${ }^{66}$. However, CGM is expensive and is not routinely available in countries threatened by malaria. It is unlikely that CGM will be the first-line investigation in such countries unless the value of CGM is established beyond doubt and the price of CGM application and consumables reduces through economies of scale.

\section{Problems and barriers to use of CGM}

We have outlined the situations in which CGM is used for children with hypoglycaemia and discussed the positive impact it can have upon disease monitoring and even therapeutics. We have previously discussed the issue of accuracy of CGM at hypoglycaemia and here we will outline the other barriers to use of CGM in disorders of hypoglycaemia.

\section{Lag time}

The lack of reliability of CGM at hypoglycaemia is not only due to poor point accuracy but also relates to the issue of $\operatorname{lag}^{70}$. Lag relates to the time delay between a change in plasma glucose and the reporting of this change by the device. Lag time is comprised of 1) Physiological lag due to the time required for glucose to diffuse from capillaries to the interstitium and is affected by skin blood flow, 2) Sensor reaction time related to the time required for glucose to diffuse from the interstitium into the sensor itself and 3) Sensor signal processing including the moving average filter and an artefact filter to allow data smoothing ${ }^{5}$.

The issue of lag time can result in significant under or overestimation of plasma glucose. When glucose is falling rapidly, lag is large and glucose can be overestimated by as much as $2.2 \mathrm{mmol} / \mathrm{L}^{71}$ resulting in non-treatment of hypoglycaemia. However, this mismatch is highly variable and therefore cannot be reliably corrected by current device algorithms. Lag time can vary from 8-40 minutes depending on the device used, level of glucose, rate of change and skin blood flow ${ }^{71}$. Fortuitously, lag time problems may actually be beneficial in the identification of hypoglycaemia in 
$\mathrm{HH}$ patient population where a spike in insulin and subsequent rapid drop in interstitial glucose is earlier than a slower subsequent decrease in plasma glucose. The lag on the device may, therefore, result in a more accurate reporting of tissue glucose than if there was no $\operatorname{lag}^{71}$. This may account for the lack of perceived lag reported in some in vivo studies ${ }^{72}$.

\section{Alarms}

As previously discussed, one of the primary benefits of CGM is its ability to predict an upcoming hypoglycaemia event using trend information. However, this prediction is only possible if device algorithms are accurate enough to alarm at appropriate times. False-positive and negative rates of 0 $100 \%$ can be achieved by altering the alarm threshold on a device ${ }^{25}$. Alarms on CGM devices do help to reduce hypoglycaemia episodes in those with diabetes ${ }^{73}$ particularly when these alarms are predictive rather than reactive ${ }^{39}$. However, alarm fatigue is a significant problem in those who use $\mathrm{CGM}^{74}$ : Patients with hypoglycaemia overnight only wake to $29 \%$ of alarms and parents of children with hypoglycaemia only wake to $37 \%{ }^{75}$. Furthermore, predictive alarms are based upon the linear correlations of glucose, carbohydrate and insulin in patients with diabetes over short periods and have not been designed for patients with $\mathrm{HH}$ who have more unpredictable hypoglycaemia events. Most devices do not contain predictive alarms and rely upon the user to identify impending hypoglycaemia using their judgement to make manual adjustments ${ }^{76}$. Herein lies the potential to automate a glucose correction response to continuous glucose sensing to develop a closed-loop system for childhood hypoglycaemia.

\section{Fingerprick testing}

Historically CGM devices have required user calibration via the measurement of fingerpick glucose using a point of care glucometer. While many devices can now self-calibrate, some still require fingerprick calibration and this is a major patient complaint ${ }^{1}$ and further source of potential inaccuracy as glucometers used for calibration vary widely and MARD can be as high as $17 \%$ in certain devices ${ }^{77}$. The number of fingerprick tests can be reduced to once a day or less when calibration tools are implemented ${ }^{32}$. The most recent devices such as the Dexcom G6 and Freestyle 
Libre are calibrated at production, obviating the need for post-insertion fingerprick plasma glucose calibrations.

\section{Clinician inertia and usability}

Physicians are considered one of the major barriers to wider adoption of $\mathrm{CGM}^{76}$. Adoption of new technology by clinicians requires a significant investment in time and training for something that is likely to benefit only a small number of their patients. There is very little in the way of formal training available for clinicians using CGM particularly amongst those working with children with non-diabetic hypoglycaemia. There is also a need for continued improvement in the usability of CGM. This is related not only to the speed of connectivity between the sensor and the receiver/cloud storage device, but also the sites at which CGM is worn on the skin - which in neonates is limited, and the length of time that they can be worn. The Advanced Technologies and Treatments for Diabetes (ATTD) Congress devised a consensus recommendation for CGM data utilisation to try to aim interpretation and standardise use ${ }^{78}$. The development of an automated interpretation of CGM data specific to those with hypoglycaemia disorders would go a long way to reducing this particular barrier to wider adoption of CGM.

\section{Cost}

In the United Kingdom, CGM is available under certain conditions for those with diabetes. In countries where individuals rely on health insurance to provide cover this situation is even more complicated and access more limited. No CGM manufacturer has a licence for use in hypoglycaemia disorders and correspondingly these are either paid for by patients or individual healthcare departments, usually only for investigative or research purposes. In low-income countries, the cost of CGM is completely prohibitive for all but the most well-off patients ${ }^{66}$. 


\section{The future for hypoglycaemia prediction}

\section{CGM developments}

Current CGM device manufacturers are focused on the use of CGM in patients with Type I and Type II Diabetes Mellitus with current development focusing on expansion to include pregnant women and ease of use for these patient groups. Sensors are likely to be further miniaturised with advancing technology and therefore become less invasive. For example, there are future plans to integrate CGM into contact lenses ${ }^{79}$ and mouth guards ${ }^{70}$ to measure tear and salivary glucose respectively as surrogates of interstitial glucose.

\section{Predictive blood glucose algorithms}

Patients have very different physiologies regarding glucose metabolism ${ }^{80}$ and factors affecting blood glucose include but are not limited to: long and short term blood glucose history, insulin secretion/administration, physical activity, dietary intake, body mass index (BMI), stress level, amount of sleep, presence of illness, medications, smoking, menstruation, allergies and altitude ${ }^{81}$. CGM devices cannot currently process any of these factors to account for variability related to lifestyle choices.

There are three main types of blood glucose prediction algorithms: Physiological, Data-Driven and Hybrid (Table 2) ${ }^{80}$. These algorithms often use a frequentist approach (regression analysis) to predict a single value but more recently a Bayesian approach (via Machine Learning, Artificial Neural Networks and Deep Learning) has been taken, resulting in a probability distribution for outcomes rather than a single value. Outcomes of all of these algorithms can either be a continuous glucose value or a classification such as the risk of hypoglycaemia ${ }^{80}$ or the certainty/uncertainty of hypoglycaemia. Algorithms with a classification outcome are not suitable for projects such as the artificial pancreas where a continuous variable is required to calculate insulin delivery rate. However, they can provide significant benefit in the prediction of hypoglycaemia in children. 
Early attempts at using CGM values to predict future continuous blood glucose values proved very inaccurate: Pappada et al demonstrated an MARD of $22 \%$ and a hypoglycaemia detection rate of only $2 \%$ using neural network algorithms ${ }^{82}$. Significant improvements have been made with Contreras et al achieving an MARD of $12 \%$ and employing a glucose specific cost function which takes in to account the clinical harmfulness of deviations from euglycaemia ${ }^{83}$. Most recently Li et al used deep neural networks to achieve an MARD of $5 \%$ and $10 \%$ when their algorithm was tested on simulated and real patient data respectively ${ }^{84}$. Due to large inter-patient variability, those algorithms which incorporated an element of personalisation improved their performance significantly ${ }^{85}$.

Predictive algorithms work better in one specific circumstance than another ${ }^{81}$ and therefore algorithms predicting continuous variables often have a poor accuracy at detecting hypoglycaemia. Jensen et al developed a model to predict the incidence of nocturnal hypoglycaemia and, despite a long prediction horizon (PH) of 3-6 hours achieved a Receiver Operating Characteristic (ROC) Area Under the Curve (AUC) of 0.79 (equivalent to an algorithm correctly categorising independent variables $79 \%$ of the time) and a negative predictive value of $97 \%^{86}$. Seo et al used a simple model with a PH of 30 minutes to predict postprandial hypoglycaemia with an AUC of 0.97 and high sensitivity and specificity of $89 \%$ and $91 \%$ respectively ${ }^{87}$.

Recent studies have combined continuous value prediction with classification algorithms. MosqueraLopez et al devised a generalised classification model and applied individualised smoothing to improve hypoglycaemia prediction from $80 \%$ to $90 \%{ }^{88}$. Their continuous value prediction resulted in 99\% of values in Zone A on an MCEG. While this was calculated with CGM values instead of fingerprick or laboratory plasma glucose values, the authors demonstrated the superiority of their predictions over that of Dexcom G6, highlighting the potential benefit of individualised algorithms ${ }^{88}$. Vehi et al have recently developed an integrated system involving a classification approach for hypoglycaemia detection as well as a continuous variable prediction to allow the user to respond appropriately to a hypoglycaemia event. This not only allows a more personalised response to hypoglycaemia but reduces the chance of an undetected event ${ }^{89}$. 
The potential for computer-generated algorithms to improve the detection of hypoglycaemia is significant but requires considerable and robust real-life applications. Few algorithms to date haveincorporated stress, medication or illness scenarios into their predictions; most studies compare predictions with CGM values rather than a gold standard and the majority only calculate MARD rather than the ability to predict hypoglycaemia. Furthermore, most algorithms are tested on simulated or historical patient data and not in patients, in real-time, and in real-life situations. Clearly, rigorous testing must be performed if predictive algorithms are to form a part of hypoglycaemia prediction and prevention clinical strategies in children with hypoglycaemia.

\section{Conclusions}

Detection of glucose levels at home has undergone significant changes over the last 100 years. Use of CGM is revolutionising the care of patients with diabetes and demonstrating significant improvements in clinical outcomes. However, the accuracy of CGM at hypoglycaemia remains poor and the efficacy of its use in disorders of hypoglycaemia in children is at best suboptimal. Small case series highlight the potential benefit of CGM in the management of these children but there are multiple barriers to regular clinical use. The use of computer-generated predictive algorithms which make use of CGM values is likely to form a significant part of hypoglycaemia prediction in the near future but will require investment and incentivisation by industry. 


\section{References}

1. Olczuk D, Priefer R. A history of continuous glucose monitors (CGMs) in self-monitoring of diabetes mellitus. Diabetes Metab Syndr Clin Res Rev. 2018;12(2):181-187. doi:10.1016/j.dsx.2017.09.005

2. Karam JH, Lorenzi $\mathrm{M}$, Young $\mathrm{CW}$, et al. Feedback-controlled dextrose infusion during surgical management of insulinomas. Am J Med. 1979;66(4):675-680. doi:10.1016/00029343(79)91183-5

3. Ginsberg BH. The FDA panel advises approval of the first continuous glucose sensor. Diabetes Technol Ther. 1999;1(2):203-204. doi:10.1089/152091599317431

4. Garg SK, Akturk HK. A new era in continuous glucose monitoring: Food and drug administration creates a new category of factory-calibrated nonadjunctive, interoperable class ii medical devices. Diabetes Technol Ther. 2018;20(6):391-394.

doi:10.1089/dia.2018.0142

5. Klonoff DC, Ahn D, Drincic A. Continuous glucose monitoring: A review of the technology and clinical use. Diabetes Res Clin Pract. 2017;133:178-192. doi:10.1016/j.diabres.2017.08.005

6. Shah R, McKinlay CJD, Harding JE. Neonatal hypoglycemia: Continuous glucose monitoring. Curr Opin Pediatr. 2018;30(2):204-208. doi:10.1097/MOP.0000000000000592

7. Kanaka-Gantenbein C. Hypoglycemia in childhood: Long-term effects. In: Pediatric Endocrinology Reviews. Vol 1. ; 2004:530-536.

8. Lucas A, Morley R, Cole TJ. Adverse Neurodevelopmental Outcome Of Moderate Neonatal Hypoglycaemia. Vol 297.; 1988.

9. Duvanel $\mathrm{CB}$, Fawer CL, Colling J, Hohlfeld P, Matthieu JM. Long-term effects of neonatal hypoglycemia on brain growth and psychomotor development in small-for-gestational-age preterm infants. J Pediatr. 1999;134(4):492-498. doi:10.1016/S0022-3476(99)70209-X

10. Burns CM, Rutherford MA, Boardman JP, Cowan FM. Patterns of cerebral injury and neurodevelopmental outcomes after symptomatic neonatal hypoglycemia. Pediatrics. 2008;122(1):65-74. doi:10.1542/peds.2007-2822

11. Lord K, Radcliffe J, Gallagher PR, Adzick NS, Stanley CA, De León DD. High risk of diabetes and neurobehavioral deficits in individuals with surgically treated hyperinsulinism. J Clin Endocrinol Metab. 2015;100(11):4133-4139. doi:10.1210/jc.2015-2539

12. Avatapalle HB, Banerjee I, Shah S, et al. Abnormal neurodevelopmental outcomes are common in children with transient congenital hyperinsulinism. Front Endocrinol (Lausanne). 2013;4(MAY). doi:10.3389/fendo.2013.00060

13. Christesen HT, Brusgaard K, Hussain K. Recurrent spontaneous hypoglycaemia causes loss of neurogenic and neuroglycopaenic signs in infants with congenital hyperinsulinism. Clin Endocrinol (Oxf). 2012;76(4):548-554. doi:10.1111/j.1365-2265.2011.04250.x

14. Gill G V., Woodward A, Casson IF, Weston PJ. Cardiac arrhythmia and nocturnal hypoglycaemia in type 1 diabetes-the "dead in bed" syndrome revisited. Diabetologia. 2009;52(1):42-45.

15. Kovatchev BP. Hypoglycemia Reduction and Accuracy of Continuous Glucose Monitoring. Diabetes Technol Ther. 2015;17(8):530-533. doi:10.1089/dia.2015.0144

16. Rodbard D. Continuous Glucose Monitoring: A Review of Recent Studies Demonstrating Improved Glycemic Outcomes. Diabetes Technol Ther. 2017;19(S3):S25-S37. doi:10.1089/dia.2017.0035

17. Van Beers CAJ, Devries JH. Continuous Glucose Monitoring: Impact on Hypoglycemia. J Diabetes Sci Technol. 2016;10(6):1251-1258. doi:10.1177/1932296816653411

18. Tomotaki S, Toyoshima K, Shimokaze T, Kawai M. Reliability of real-time continuous glucose monitoring in infants. Pediatr Int. July 2019. doi:10.1111/ped.13961

19. Perri A, Giordano L, Corsello M, et al. Continuous glucose monitoring (CGM) in very low birth weight newborns needing parenteral nutrition: Validation and glycemic percentiles. Ital J Pediatr. 2018;44(1). doi:10.1186/s13052-018-0542-5

20. Dexcom G6 CGM System User Guide, 2018.

21. Data on File, Abbott Diabetes Care Inc. Clinical Report: Evaluation of the Accuracy of the 
Abbott Sensor-Based Interstitial Glucose Monitoring System 2014.

22. Monsod TP, Flanagan DE, Rife F, et al. Do sensor glucose levels accurately predict plasma glucose concentrations during hypoglycemia and hyperinsulinemia? Diabetes Care. 2002;25(5):889-893. doi:10.2337/diacare.25.5.889

23. Reiterer F, Polterauer $P$, Schoemaker $M$, et al. Significance and Reliability of MARD for the Accuracy of CGM Systems. J Diabetes Sci Technol. 2017;11(1):59-67.

doi:10.1177/1932296816662047

24. Pardo S, Simmons DA. The Quantitative Relationship Between ISO 15197 Accuracy Criteria and Mean Absolute Relative Difference (MARD) in the Evaluation of Analytical Performance of Self-Monitoring of Blood Glucose (SMBG) Systems. J Diabetes Sci Technol.

2016;10(5):1182-1187. doi:10.1177/1932296816644468

25. Noujaim SE, Horwitz D, Sharma M, Marhoul J. Accuracy requirements for a hypoglycemia detector: An analytical model to evaluate the effects of bias, precision, and rate of glucose change. J Diabetes Sci Technol. 2007;1(5):652-668. doi:10.1177/193229680700100509

26. Shah R, McKinlay CJD, Harding JE. Neonatal hypoglycemia: Continuous glucose monitoring. Curr Opin Pediatr. 2018;30(2):204-208. doi:10.1097/MOP.0000000000000592

27. Conrad SC, Mastrototaro JJ, Gitelman SE. The use of a continuous glucose monitoring system in hypoglycemic disorders. J Pediatr Endocrinol Metab. 2004;17(3):281-288. doi:10.1515/JPEM.2004.17.3.281

28. Signal M, Le Compte A, Harris DL, Weston PJ, Harding JE, Chase JG. Impact of retrospective calibration algorithms on hypoglycemia detection in newborn infants using continuous glucose monitoring. Diabetes Technol Ther. 2012;14(10):883-890. doi:10.1089/dia.2012.0111

29. King C, Anderson SM, Breton M, Clarke WL, Kovatchev BP. Modeling of Calibration Effectiveness and Blood-to-Interstitial Glucose Dynamics as Potential Confounders of the Accuracy of Continuous Glucose Sensors during Hyperinsulinemic Clamp. J Diabetes Sci Technol. 2007;1(3):317-322. doi:10.1177/193229680700100302

30. Guerra S, Facchinetti A, Sparacino G, Nicolao G De, Cobelli C. Enhancing the accuracy of subcutaneous glucose sensors: A real-time deconvolution-based approach. IEEE Trans Biomed Eng. 2012;59(6):1658-1669. doi:10.1109/TBME.2012.2191782

31. Zavitsanou S, Lee JB, Pinsker JE, Church MM, Doyle FJ, Dassau E. A Personalized Week-toWeek Updating Algorithm to Improve Continuous Glucose Monitoring Performance. In: Journal of Diabetes Science and Technology. Vol 11. SAGE Publications Inc.; 2017:1070-1079. doi:10.1177/1932296817734367

32. Acciaroli G, Vettoretti M, Facchinetti A, Sparacino G. Toward Calibration-Free Continuous Glucose Monitoring Sensors: Bayesian Calibration Approach Applied to Next-Generation Dexcom Technology. Diabetes Technol Ther. 2018;20(1):59-67. doi:10.1089/dia.2017.0297

33. De Block C, Manuel-Y-keenoy B, Van Gaal L. A review of current evidence with continuous glucose monitoring in patients with diabetes. In: Journal of Diabetes Science and Technology. Vol 2. ; 2008:718-727. doi:10.1177/193229680800200426

34. Bergenstal RM, Tamborlane W V., Ahmann A, et al. Effectiveness of sensor-augmented insulin-pump therapy in type 1 diabetes. N Engl J Med. 2010;363(4):311-320. doi:10.1056/NEJMoa1002853

35. Wentholt IME, Hoekstra JBL, Devries JH. Continuous glucose monitors: The long-awaited watch dogs? Diabetes Technol Ther. 2007;9(5):399-409. doi:10.1089/dia.2007.0215

36. Langendam M, Luijf YM, Hooft L, Devries JH, Mudde AH, Scholten RJPM. Continuous glucose monitoring systems for type 1 diabetes mellitus. Cochrane Database Syst Rev. 2012;2017(12). doi:10.1002/14651858.CD008101.pub2

37. van Beers CAJ, DeVries JH, Kleijer SJ, et al. Continuous glucose monitoring for patients with type 1 diabetes and impaired awareness of hypoglycaemia (IN CONTROL): a randomised, open-label, crossover trial. Lancet Diabetes Endocrinol. 2016;4(11):893-902. doi:10.1016/S2213-8587(16)30193-0

38. Battelino T, Phillip M, Bratina N, Nimri R, Oskarsson P, Bolinder J. Effect of continuous glucose monitoring on hypoglycemia in type 1 diabetes. Diabetes Care. 2011;34(4):795-800. doi:10.2337/dc10-1989 
39. Puhr S, Derdzinski M, Welsh JB, Parker AS, Walker T, Price DA. Real-World Hypoglycemia Avoidance with a Continuous Glucose Monitoring System's Predictive Low Glucose Alert. Diabetes Technol Ther. 2019;21(4):155-158. doi:10.1089/dia.2018.0359

40. Beardsall K, Ogilvy-Stuart AL, Ahluwalia J, Thompson M, Dunger DB. The continuous glucose monitoring sensor in neonatal intensive care. Arch Dis Child Fetal Neonatal Ed. 2005;90(4):307-310. doi:10.1136/adc.2004.051979

41. Grunau RE, Whitfield MF, Petrie-Thomas J, et al. Neonatal pain, parenting stress and interaction, in relation to cognitive and motor development at 8 and 18 months in preterm infants. Pain. 2009;143(1-2):138-146. doi:10.1016/j.pain.2009.02.014

42. Harris DL, Battin MR, Weston PJ, Harding JE. Continuous glucose monitoring in newborn babies at risk of hypoglycemia. J Pediatr. 2010;157(2):198-202.e1.

doi:10.1016/j.jpeds.2010.02.003

43. Uettwiller F, Chemin A, Bonnemaison E, Favrais G, Saliba E, Labarthe F. Real-time continuous glucose monitoring reduces the duration of hypoglycemia episodes: A randomized trial in very low birth weight neonates. PLoS One. 2015;10(1). doi:10.1371/journal.pone.0116255

44. Thomson L, Elleri D, Bond S, Howlett J, Dunger DB, Beardsall K. Targeting glucose control in preterm infants: Pilot studies of continuous glucose monitoring. Arch Dis Child Fetal Neonatal Ed. 2019;104(4):F353-F359. doi:10.1136/archdischild-2018-314814

45. McKinlay CJD, Alsweiler JM, Ansell JM, et al. Neonatal glycemia and neurodevelopmental outcomes at 2 years. N Engl J Med. 2015;373(16):1507-1518. doi:10.1056/NEJMoa1504909

46. Kishnani PS, Austin SL, Abdenur JE, et al. Diagnosis and management of glycogen storage disease type I: A practice guideline of the American College of Medical Genetics and Genomics. Genet Med. 2014;16(11):1-29. doi:10.1038/gim.2014.128

47. Hershkovitz E, Rachmel A, Ben-Zaken H, Phillip M. Continuous glucose monitoring in children with glycogen storage disease type I. J Inherit Metab Dis. 2001;24(8):863-869. doi:10.1023/A:1013996325720

48. Maran A, Crepaldi C, Avogaro A, et al. Continuous glucose monitoring in conditions other than diabetes. Diabetes Metab Res Rev. 2004;20(SUPPL. 2):50-55. doi:10.1002/dmrr.518

49. Kasapkara CS, Cinasal Demir G, Hasanoğlu A, Tümer L. Continuous glucose monitoring in children with glycogen storage disease type i. Eur J Clin Nutr. 2014;68(1):101-105. doi:10.1038/ejcn.2013.186

50. Herbert M, Pendyal S, Rairikar M, Halaby C, Benjamin RW, Kishnani PS. Role of continuous glucose monitoring in the management of glycogen storage disorders. J Inherit Metab Dis. 2018;41(6):917-927. doi:10.1007/s10545-018-0200-5

51. White FJ, Jones SA. The use of continuous glucose monitoring in the practical management of glycogen storage disorders. J Inherit Metab Dis. 2011;34(3):631-642. doi:10.1007/s10545011-9335-3

52. Bornstein SR, Allolio $B$, Arlt $W$, et al. Diagnosis and treatment of primary adrenal insufficiency: An endocrine society clinical practice guideline. J Clin Endocrinol Metab. 2016;101(2):364389. doi:10.1210/jc.2015-1710

53. Watanabe T, Ozawa A, Ishii S, et al. Usage of continuous glucose monitoring (CGM) for detecting an unrecognized hypoglycemia and management of glucocorticoid replacement therapy in adult patients with central hypoadrenalism. Endocr J. 2018;65(5):547-556. doi:10.1507/endocrj.EJ16-0387

54. Cambiaso P, Schiaffini R, Pontrelli G, et al. Nocturnal hypoglycaemia in ACTH and GH deficient children: Role of continuous glucose monitoring. Clin Endocrinol (Oxf). 2013;79(2):232-237. doi:10.1111/cen.12123

55. Menni F, Pascale De Lonlay ;, Sevin C, et al. Neurologic Outcomes of 90 Neonates and Infants With Persistent Hyperinsulinemic Hypoglycemia. Vol 107.; 2001.

56. Saif M, Kapoor A, Kochar I, Jindal R. Continuous glucose monitoring system for congenital hyperinsulinemia. Indian Pediatr. 2013;50(4):421-422. doi:10.1007/s13312-013-0103-3

57. Philippon $M$, Séjil $S$, Mugnier $M$, et al. Use of the continuous glucose monitoring system to treat insulin autoimmune syndrome: Quantification of glucose excursions and evaluation of treatment efficacy. Diabet Med. 2014;31(7):e20-e24. doi:10.1111/dme.12418 
58. Alsaffar H, Turner L, Yung Z, Didi M, Senniappan S. Continuous Flash Glucose Monitoring in children with Congenital Hyperinsulinism; first report on accuracy and patient experience. Int J Pediatr Endocrinol. 2018;2018(1). doi:10.1186/s13633-018-0057-2

59. Vezzosi D, Guillaume E, Bennet A, Mouly C, Hanaire H, Caron P. Medical therapy in patients with endogenous hypoglycaemia: Is euglycaemia achievable? Clin Endocrinol (Oxf). 2019;90(6):798-804. doi:10.1111/cen.13961

60. Munir A, Choudhary P, Harrison B, Heller S, Newell-Price J. Continuous glucose monitoring in patients with insulinoma. Clin Endocrinol (Oxf). 2008;68(6):912-918. doi:10.1111/j.13652265.2007.03161.x

61. Sawyer AM, Schade DS. Use of a continuous glucose monitor in the management of inoperable meta static insulinoma: A case report. Endocr Pract. 2008;14(7):880-883. doi:10.4158/EP.14.7.880

62. Okabayashi T, Shima Y, Sumiyoshi T, et al. Diagnosis and management of insulinoma. World J Gastroenterol. 2013;19(6):829-837. doi:10.3748/wjg.v19.i6.829

63. Manabe $M$, Morimatsu $H$, Egi $M$, et al. [Anesthetic management of pediatric patients with insulinoma using continuous glucose monitoring]. Masui. 2009;58(6):757-759. http://www.ncbi.nlm.nih.gov/pubmed/19522271. Accessed December 5, 2019.

64. Rodríguez Flores M, Cruz Soto RC, Vázquez Velázquez V, Soriano Cortés RR, Aguilar Salinas C, García García E. Continuous glucose monitoring in the management of patients after gastric bypass. Endocrinol Diabetes Metab Case Reports. 2019;2019. doi:10.1530/edm-18-0155

65. Nielsen JB, Abild CB, Pedersen AM, Pedersen SB, Richelsen B. Continuous Glucose Monitoring After Gastric Bypass to Evaluate the Glucose Variability After a Low-Carbohydrate Diet and to Determine Hypoglycemia. Obes Surg. 2016;26(9):2111-2118. doi:10.1007/s11695-016-2058-7

66. Bila R, Varo R, Madrid L, Sitoe A, Bassat Q. Continuous glucose monitoring in resourceconstrained settings for hypoglycaemia detection: Looking at the problem from the other side of the coin. Biosensors. 2018;8(2):2-7. doi:10.3390/bios8020043

67. Osier FHA, Berkley JA, Ross A, Sanderson F, Mohammed S, Newton CRJC. Abnormal blood glucose concentrations on admission to a rural Kenyan district hospital: Prevalence and outcome. Arch Dis Child. 2003;88(7):621-625. doi:10.1136/adc.88.7.621

68. Jallow M, Casals-Pascual C, Ackerman H, et al. Clinical Features of Severe Malaria Associated with Death: A 13-Year Observational Study in The Gambia. PLoS One. 2012;7(9). doi:10.1371/journal.pone.0045645

69. Willcox ML, Forster M, Dicko MI, Graz B, Mayon-White R, Barennes H. Blood glucose and prognosis in children with presumed severe malaria: Is there a threshold for "hypoglycaemia"? Trop Med Int Heal. 2010;15(2):232-240. doi:10.1111/j.13653156.2009.02444.x

70. Chen C, Zhao XL, Li ZH, Zhu ZG, Qian SH, Flewitt AJ. Current and emerging technology for continuous glucose monitoring. Sensors (Switzerland). 2017;17(1). doi:10.3390/s17010182

71. Davey RJ, Low C, Jones TW, Fournier PA. Contribution of an intrinsic lag of continuous glucose monitoring systems to differences in measured and actual glucose concentrations changing at variable rates in vitro. J Diabetes Sci Technol. 2010;4(6):1393-1399.

doi:10.1177/193229681000400614

72. Caplin NJ, O'Leary P, Bulsara M, Davis EA, Jones TW. Subcutaneous glucose sensor values closely parallel blood glucose during insulin-induced hypoglycaemia. Diabet Med. 2003;20(3):238-241. doi:10.1046/j.1464-5491.2003.00837.x

73. Davey RJ, Jones TW, Fournier PA. Effect of short-term use of a continuous glucose monitoring system with a real-time glucose display and a low glucose alarm on incidence and duration of hypoglycemia in a home setting in type 1 diabetes mellitus. J Diabetes Sci Technol. 2010;4(6):1457-1464. doi:10.1177/193229681000400620

74. Pickup JC, Holloway MF, Samsi K. Real-time continuous glucose monitoring in type 1 diabetes: A qualitative framework analysis of patient narratives. Diabetes Care. 2015;38(4):544-550. doi:10.2337/dc14-1855

75. Buckingham B, Block J, Burdick J, et al. Response to nocturnal alarms using a real-time glucose sensor. Diabetes Technol Ther. 2005;7(3):440-447. doi:10.1089/dia.2005.7.440 
76. Rodbard D. Continuous Glucose Monitoring: A Review of Successes, Challenges, and Opportunities. Diabetes Technol Ther. 2016;18(S2):S23-S213. doi:10.1089/dia.2015.0417

77. Dunne N, Viggiani MT, Pardo S, Robinson C, Parkes JL. Accuracy Evaluation of CONTOUR ${ }^{\oplus}$ PLUS Compared With Four Blood Glucose Monitoring Systems. Diabetes Ther. 2015;6(3):377-388. doi:10.1007/s13300-015-0121-3

78. Battelino T, Danne T, Bergenstal RM, et al. Clinical targets for continuous glucose monitoring data interpretation: Recommendations from the international consensus on time in range. Diabetes Care. 2019;42(8):1593-1603.

79. Ascaso FJ. Noninvasive Continuous Monitoring of Tear. Optom Vis Sci. 2016;93(4):426-434. doi:10.1097/OPX.0000000000000698

80. Oviedo S, Vehí J, Calm R, Armengol J. A review of personalized blood glucose prediction strategies for T1DM patients. Int $j$ numer method biomed eng. 2017;33(6). doi:10.1002/cnm.2833

81. Woldaregay AZ, Årsand E, Walderhaug S, et al. Data-driven modeling and prediction of blood glucose dynamics: Machine learning applications in type 1 diabetes. Artif Intell Med. 2019;98(August 2018):109-134. doi:10.1016/j.artmed.2019.07.007

82. Pappada SM, Cameron BD, Rosman PM, et al. Neural network-based real-time prediction of glucose in patients with insulin-dependent diabetes. Diabetes Technol Ther. 2011;13(2):135141. doi:10.1089/dia.2010.0104

83. Contreras I, Oviedo S, Vettoretti M, Visentin R, Vehí J. Personalized blood glucose prediction: A hybrid approach using grammatical evolution and physiological models. PLoS One. 2017;12(11). doi:10.1371/journal.pone.0187754

84. Li K, Liu C, Zhu T, Herrero P, Georgiou P. GluNet: A Deep Learning Framework For Accurate Glucose Forecasting. IEEE J Biomed Heal Informatics. 2019;PP(c):1-1. doi:10.1109/jbhi.2019.2931842

85. Li J, Fernando C. Smartphone-based personalized blood glucose prediction. ICT Express. 2016;2(4):150-154. doi:10.1016/j.icte.2016.10.001

86. Jensen MH, Dethlefsen C, Vestergaard P, Hejlesen O. Prediction of Nocturnal Hypoglycemia From Continuous Glucose Monitoring Data in People With Type 1 Diabetes: A Proof-ofConcept Study. J Diabetes Sci Technol. 2019. doi:10.1177/1932296819868727

87. Seo W, Lee Y Bin, Lee S, Jin SM, Park SM. A machine-learning approach to predict postprandial hypoglycemia. BMC Med Inform Decis Mak. 2019;19(1). doi:10.1186/s12911019-0943-4

88. Mosquera-Lopez C, Dodier R, Tyler N, Resalat N, Jacobs P. Leveraging a Big Dataset to Develop a Recurrent Neural Network to Predict Adverse Glycemic Events in Type 1 Diabetes. IEEE J Biomed Heal Informatics. 2019;PP(XX):1-1. doi:10.1109/jbhi.2019.2911701

89. Vehí J, Contreras I, Oviedo S, Biagi L, Bertachi A. Prediction and prevention of hypoglycaemic events in type-1 diabetic patients using machine learning. Health Informatics J. June 2019:1460458219850682. doi:10.1177/1460458219850682 
Figure 1. Mechanism of action of a Continuous Glucose Monitoring device. Diagrams show from left to right an increasing level of detail from the patient to the chemical reaction. Patients attach a sensor to their skin which automatically inserts a subcutaneous needle. This resides in the interstitial space and measures glucose concentrations. Via Glucose oxidase the sensor generates an electrical current proportional to the glucose concentration in the interstitium. This electrical current is passed through a continuously shifting algorithm to generate a glucose value which is transmitted wirelessly to the receiving unit or compatible smartphone for display to the patient.

Figure 2. A Modified Clarke Error Grid (MCEG). An MCEG can be used to compare clinical accuracy of values between a reference method and a new device. Values in Zone A will lead to clinically correct decisions being made. Values in Zone E represent a significant and dangerous over or underestimate of the true value and have the potential to trigger the opposite treatment to that required. Values in Zone $D$ are unlikely to trigger the opposite treatment to that required but more likely a lack of treatment (e.g. a glucose bolus may not be given to a child in which it is required). Values in Zone $C$ are at risk of triggering an appropriate response but likely at a lesser magnitude than required. Values in Zone B will trigger clinical decisions that are not correct but are uncritical. Devices with more values in Zone A will offer a higher clinical accuracy than those with values in other areas.

\begin{tabular}{|l|l|l|l|l|}
\hline & Dexcom G6 & Freestyle Libre & Medtronic Guardian & Medtrum A6 \\
\hline Calibrations & None & None & 2/day (min) & 2/day (min) \\
\hline Warmup & 2 hours & 1 hour & 2 hours & 2 hours \\
\hline Wear length & 10 days & 14 days & 7 days & 14 days \\
\hline MARD & $9 \%$ & $11 \%$ & $9 \%$ & $9 \%$ \\
\hline Low alarms? & Yes & No & Yes & Yes \\
\hline Age limit & 2 years + & 4 years + & 14 years + & 2 years + \\
\hline
\end{tabular}

Table 1. Comparisons of four common CGM devices licenced for use in children. Calibrations relate to the minimum fingerprick calibrations per day recommended by the manufacturers. Warmup time relates to the time from insertion of the device to when it begins to provide glucose readings. MARD = Mean Absolute Relative Difference and is a commonly reported accuracy measure. MARD values quoted here are those reported by the individual manufacturers. Low alarms? relates to whether the device provides an auditory alarm if blood sugar drops below a certain value. Information all obtained from manufacturer websites.

\begin{tabular}{|l|l|}
\hline $\begin{array}{l}\text { Type of } \\
\text { Algorithm }\end{array}$ & Description \\
\hline Physiological & $\begin{array}{l}\text { Model blood glucose dynamics based on a comprehensive knowledge of the } \\
\text { patient's physiology. These models require extensive expert input to create but } \\
\text { can then be used without any prior patient data. }\end{array}$ \\
\hline Data Driven & $\begin{array}{l}\text { These algorithms use data mining from large CGM datasets and regression analysis } \\
\text { to provide a mathematical prediction of future glucose trends (known as a black- } \\
\text { box approach). This requires less expert input initially but is dependent upon the } \\
\text { availability of existing datasets and assumes similarities between those patients } \\
\text { using the algorithm for prediction and those from whom the initial data was taken. }\end{array}$ \\
\hline Hybrid & $\begin{array}{l}\text { These models combine physiological knowledge with data-driven regression to } \\
\text { produce a complex predictive algorithm. }\end{array}$ \\
\hline
\end{tabular}


Table 2. Description of the three main types of blood glucose predictive algorithm. Different approaches are taken towards the prediction of blood glucose values, with each approach benefiting from a different strength. Multiple examples of each type of algorithm are available and vary widely in their predictive accuracy. Outcomes of all three types of algorithm can either be as a continuous glucose value or as a binary classification e.g.s hypoglycaemia or not. 